\title{
COMET DATA COLLECTIONS
}

"H. L. Giclas

The question of the present day usefulness of the great wealth of observational material on comets buried in the archives of the older observatories is an important problem that should be evaluated soon if any action is to be taken before much of it is lost or destroyed. The beginning step has been taken with the publication of the isophotometric atlas and the Atlas of Cometary Forms which is a direct result of Recommendation No. 1 of Commission 15 of the IAU meeting at Prague in 1967. At that time a repository for cometary photographs and spectrograms was also discussed. However, in the light of observational advances and the present capability of quantitative correlation of structural changes with conderisations or annomalies of the solar plasma, it is desirable to establish again that this old observational material may be useful.

Since the greatest structural changes cccur nearest the sun where observations are most difficult and time limited, observations made at ground based telescopes at different longitudes contiguous in time yield the only link to the continuity of developments. Perhaps the greatest argument for collecting the earlier direct photographs and spectrograms together is the fact that the observations are unique. Though we can plan a more comprehensive observational program in the future correlating radio, OAO observations, and etc., with ground based observations, because of the unpredictable frequency of such objects, we are looking at decades of time in order to accumulate and replace this existing material. With the growing 
premium on building and record storage space at the older observatories, it is easy to rationalize a clean-up of old, little used, bulky data. Also with the death or retirement of older staff members, knowledge of what observational material may exist and location of the records are more and more difficult to recover. Therefore, in this matter, time is of the essence. For example, at the Lowell Observatory there are over 2400 direct photographs and spectrograms of 170 different comets that have accumulated since the first six photographs of Comet c 1905. To enumerate a few of the earlier comets in the file of plates:

\begin{tabular}{clc} 
No. of Plates & \multicolumn{2}{c}{ Comet } \\
27 & Giacobini & 1906 \\
59 & Morehouse & 1908 \\
57 direct, 91 spectra & Comet a & 1910 \\
334 & Halley's & 1910 \\
17 & Mellish & 1915 a \\
12 & Wolf b & 1917
\end{tabular}

Most of these plates were taken with either a 5-inch Brashear lens of 35 inches focal length or a 9-inch lens also made by Brashear of 46 inches focal length. Many of the Halley's comet photographs, when the tail was 40 to 50 degrees in length, were made with numerous different portrait lenses from 7 -inch focal length to 14-inch.

With the 13 -inch astrograph $(\mathrm{fl}=66 ! 5)$, I have taken over 1500 plates of comets since 1936. Of these about one-third are short exposure plates taken for position measurement, about 100 are unsuccessful attempts at recovery or checks which leaves around 1000 that may be useful to study structural detail. To enumerate the more extensive series: 
No. of Plates
Comet

Peltier 1936 a

Finsler

Whipple 1942

Timme rs

$1947 \mathrm{n}$

Arend-Roland

Kohoutek

Bobrovnikoff in his paper on Halley's comet (Lick Pubs. 17, Part 2) got together 438 original plates and 271 reproductions. 227 of these plates were made by H. D. Curtis on the Crossley reflector, 60 by Ferdinand E1lerman with a 6" Brashear and $21 / 4 "$ Tessar on Oahu, and 31 by Adams and Babcock with the $60^{\prime \prime}$ Mt. Wilson reflector. Add to this the 334 at Lowe11 and hundreds from other observatories and see how much more could be done. Other observatories have collections - Dr. Roemer, I am sure, has taken as many or more than anyone. Say that only one-half of the 300 observatories listed in the American Ephemeris would have some material that would be useful, one can begin to appreciate the magnitude of the task of just evaluating what might be avsilable.

At Lowell we have had some experience in making world-wide collections of data in connection with the Planetary Research Center now under the direction of W. A. Baum. It had its inception from a resolution of Commission 16 at the 1961 I.A.U. meeting at Berkeley which stated, "The committee appointed by Comm. 16 on "Internationa1 Collaboration for P1anetary Observations" desires to facilitate international collaboration on planetary studies by the eventual establishment of at least two data centers, one in the U.S.A. and one in Europe; and meanwhile requests observatories having large collections of planetary photographs to make these available for such 
studies as require a full coverage in longitude" (IAU Proceedings, 1961, 245). With support from NASA over 17,355 image sequences of planets from 27 different sources scattered all over the world and dating back to the turn of the century were collected, copied and indexed. (An image sequence can be anything from four to forty single images of a planet on a photographic plate or film). Added to this older collection of data, and again with NASA support, an International Planetary Patrol was organized and operated by Lowell Observatory from July 1968 to July 1974. This added a total of 87,234 additional image sequences $(1,200,000$ images) of the planets from eight observing stations strategically distributed around the earth in longitude to the collection. The entire plate collection is indexed and cataloged in the computer so that retrival of data may be made from any one or more of twelve different criteria.

Just a word on the effectiveness of an organized world wide patrol. The Lowell Planetary Patrol Program has produced a photographic collection of unprecedented size, homogeneity, and continuity with time. It exceeds by five times the number of image sequences in the historical file collected from all over the world and spanning the previous 60 years which itself was the largest collection of planetary photographs in the world prior to the Patrol. Examples of what a systematic world wide network has accomplished for the first time is observations of an entire revolution of Mars at approximately two-hour intervals, the development and regression of the great dust storm in 1971 at the time of the Mariner 9 encounter. Coverage was such that the progress could be followed on a near hourly basis. Also other interesting "ministorms" were covered in 1971 and 1973. Similar programs on the development and velocities of clouds on Jupiter were followed. Also from orservations of the dark markings seen on ultraviolet photographs of Venus, 
a determination of the rotational period of these features was made. Applying these few examples as an analogy one can easily see the possibilities a coordinated program of world wide comet observation might give. If it is the consensus of this group that the older existing data is useful, then an effort should be made to initiate a comprehensive survey of what material is available. From this an evaluation of the scope of the probler can be made and the next step taken. 


\section{DISCUSSION}

J. C. Brandt: I would like to second almost every word that you say. When Belton and I were gathering the material for our Comet Tail Orientation Catalogue about ten years ago we encountered about every problem that you have mentioned. Perhaps one that you didn't emphasize is that most observatory directors are very cooperative but they do not know what is in their own collections. And we found many of our things by scanning log books, when Belton and I spent many, many days just reading old observing books until we found a notation that would mention a comet.

And I can add that to the extent that that catalogue has been valuable, we have been able to utilize observations to infer solar wind and comet properties so far back to 1889 , and I feel that these old observations are exceptionally valuable, and - again, I can just second the remarks that you have made.

F. L. Whipple: I feel that this suggestion is extremely important, and I felt that we are in a very strange position, talking about comets, and this morning's discussion emphasized that we don't know what a normal comet is.

The fact is, that we have not proceeded to the point in comet studies of having any taxonomic study of comets. We do not have classifications of comets in any real sense. Some of the early work you mentioned there, a few years ago, was extremely valuable, and I think it would be a great contribution to the science if somebody, and about two assistants, would spend about three years going to all the observatories and studying all the spectra thoroughly, not to mention the photography, which is a bigger job.

There's a second point I'd like to make, having the floor at this time. Perhaps somebody knows the answer: whatever happened to the compilation, and I think it was a partial digest, of all of the literature on comets, that was put together by N. T. Bobrovnikov, it must have been about two decades ago? I know he had a couple of thousand pages of it, that I heard about, and I saw a microfilm of part of it, and I wonder whether that was ever put into a coherent form, and whether it is available to anyone for use, and if not, if it could be put together. Maybe NASA could make a contribution by somehow or other getting it done.

A. H. Delsemme: It happens that next week I'm going to spend two days in Columbus to make a digest of these - Bobromikov's papers. When I say digest I don't know yet what I'm going to do. I'm going to try to assess them to make a table of contents, to list the comets, et cetera, and to make them, by and large, available to the members of Commission 15, when they need them.

That's what I intend to do. I don't know yet whether I will succeed, really, but I intend at least not to let these precious papers get lost, you know. 


\section{DISCUSSION (Continued)}

B. Donn: When Giclas proposed to describe the Lowell collection, it occurred to me that this was a good issue to raise, in a more general form, what to do with all of these enormous files, and how it might be done. We ran into the problem with the comet Atlas, with Dr. Rahe going to observatories and trying to track down some of these old plates and finding out, well, sometimes you can find them if you look through the log books; sometimes the $\log$ is there but you can't find the plate and nobody knows what's happened to them.

And it also turns out if you begin looking through some of the iiterature, you find that there exist, really more sequences of comet plates than one realizes. In some of these older records there are series of plates taken on successive nights, which are just the kind of thing that the people who are now getting involved in the plasma studies, the analysis of comet tails, really need to know just how do these comet tails behave and what do they look like and what are the variations?

The idea of the Atlas was to try to get us a representative picture, but you really want to have available the details, and I think what we need to come up with is - and maybe by having this discussion early we could try to come up during the course of this week with some procedures we'll follow up and get some idea how to go about this suggestion of doing something about it.

And I think maybe some of us who are interested can get together informally and see what might be done. One possibility may be to notify the chairman of Commission 15 that they should do something about, or at least support this sort of thing.

W. Jackson: I hope somebody gets some money for it.

\section{(Laughter.)}

Looks like it's a whole lot of traveling.

D. D. Meisel: Having worked under Bobrovnikov for several years I have seen those notebooks. There are five of them, and they are complete to 1943 .

I was asked by Nicholas if I wanted them, and knowing the job of putting them together, I said diplomatically, "No, you'd better leave them at Ohio State," which he did. So they ought to be intact, and to my knowledge it resembles the Svetsviatskii stuff, only it's about three times as extensive, with all references cited, including the double references that usually are wrongly quoted. 


\section{DISCUSSION (Continued)}

So I think that - you know - it may take you three or five years to get through it, but good luck.

\section{(Laughter.)}

B. Jambor: I'd like to ask how many of these plates, mostly the ones concerning comets and the older ones, are calibrated? To do good photometry on them you would need some kind of calibration, and then if they're not that takes quite a bit of the value out of them.

\section{H. L. Giclas: Right.}

I'm just afraid that the ones at Lowell are not calibrated. We calibrated all the planetary observations with tube sensitometers, but in the case of the comets I'm afraid this has not been done, and I agree with you it loses a great deal of the value.

R. G. Roosen: Well, it's strictly a matter of value judgments and personal opinion, having taken a lot of comet plates and scratched my head over them myself, and seeing a lot of other people scratch their heads.

Basically I think there are several points that can be made. First of all, Jack Brandt has already demonstrated how simply measuring the direction of the tail is of extreme value over the years. All that the Catalogue of Comet Tail Orientations, the Belton and Brandt catalogue, does, is measure the position angle of the tail. And he's been able to demonstrate a lot of things about the solar wind.

As has been mentioned before, the morphology of the tails isn't understood at all, and just because we don't understand this morphology very well doesn't mean that we should figure that there's no value in a picture of a lot of kinks and whirls. Tomorrow I'm going to show a particular kink that has me scratching my head, and I'm sure there's a lot more of this information on these plates.

In terms of what it would take to get this information where they would be safe, obviously many of these plates, especially the older ones, are very big, very heavy, very bulky, but Klaus Jockers took about 150 of our $4 \times 5$ plates from JOCR down to Sac Peak, and in one day, he made positive film copies, 70 millimeter film copies of all of these plates, and he'll show a movie which has virtually all the resolution on the original plate.

So from my way of thinking it's not a severely difficult problem. I would guess that if the National Academy, for instance, were willing to put up money for a fellowship for one or two years, that it wouldn't be too hard to get the 


\section{DISCUSSION (Continued)}

photographic equipment together and have a single individual go a round to observatories, do all this seeking and searching that's necessary, and in about two years, with the cost of his salary, a little bit of travel and a little bit of film, we really and truly could have a library on positive film copies that would be available.

Now, the thing is, that a positive film copy is also advantageous because you can get - oh, probably 300 or 400 plates on a little piece of film that you can put in a little bitty can, so you wouldn't have to have the large storage building built for your records.

W. Jackson: Curt McCracken mentioned that in addition to the professional photographs there are amateur photographs that I guess should be included in the Comet Catalogue.

Would we like to, at this particular time, suggest criteria or set up a committee to look into this problem more completely? Is that the general consensus at this point, or do we want to just drop it as we do a lot of things and proceed, if somebody comes up with something later on?

A. H. Delsemme: Well, I think it's a very important matter that came back again and again over the years, and we have never done something really serious about it, and that's too bad.

I would like to propose that we do something. For instance, in the framework of Commission 15 it could be done. But I need, of course, advice, and -

W. Jackson: Would anybody like to volunteer -

(After much discussion a committee was appointed consisting of A. H. Delsemme (chairman IAU Commission 15), H. Giclas, J. Rahe, F. Dossin, M. Wallis, and R. Roosen. 